Appendix: Content Summaries of the Selected Best Papers for the IMIA Yearbook 2018 "Signals, Sensors, and Imaging Informatics" Section

\author{
Bote JM, Recas J, Rincon F, Atienza D, \\ Hermida R
}

A modular low-complexity ECG delineation algorithm for real-time embedded systems

IEEE J Biomed Health Inform

2018;22(2):429-41

The electrocardiogram (ECG) signal is essential to monitor the heart and evaluate its condition. Most techniques for automated ECG analysis can be computationally intensive, which limits their applicability in low-power wearable devices. The authors present a new modular, low-complexity algorithm that requires a reduced number of operations per second and smaller memory footprint to perform real-time ECG analysis on resource-constrained embedded systems. The system delineated and located the peaks and boundaries of the different ECG waves, such as the $\mathrm{P}$ wave, the QRS complex, and the $T$ wave with reduced mathematical complexity. The algorithm achieved similar or better accuracy than other more advanced and computationally intensive state-of-theart techniques, and could be useful for use in ultralow-power mobile or wearable devices.

\author{
Grossmann P, Stringfield 0, El-Hachem \\ N, Bui MM, Rios Velazquez E, Parmar C, \\ Leijenaar RT, Haibe-Kains B, Lambin P, \\ Gillies RJ, Aerts HJ
}

\section{Defining the biological basis of radiomic phenotypes in lung cancer \\ ELife 2017;6:e23421}

Radiomics is an emerging field that aims to extract specific quantitative features from medical images to define a patient's "imaging phenotype." In cancer imaging, radiomics has been associated with several clinical endpoints, but the complex relationships of radiomics features, clinical factors, and tumor biology are largely unknown. The authors identified a relationship between imaging features, immune response, inflammation, and survival, which was validated by immunohistochemical staining. Several imaging features showed predictive value for activity of specific pathways: for example, intra-tumor heterogeneity predicted RNA polymerase transcription, and intensity dispersion predicted autodegradation of an ubiquitin ligase. The model achieved the highest prognostic accuracy from the combination of radiomic, genetic, and clinical data.

\section{Larson DB, Chen MC, Lungren MP, Halabi SS, Stence NV, Langlotz CP \\ Performance of a deep-learning neural network model in assessing skeletal maturity on pediatric hand radiographs}

\section{Radiology 2018; 287(1):313-22}

Assessment of radiographic "bone age" typically has been a manual task that requires reference to a standard atlas of children's hand radiographs. The authors automated this process using a collection of more than 14,000 radiographs and corresponding clinical reports to train and test their model. They found that a convolutional neural network model can estimate skeletal maturity with an accuracy similar to that of an expert radiologist and to existing automated models. The authors' results provide an important example that suggests that deep learning models may be broadly applicable to a variety of diagnostic imaging tasks without requiring specialized subject matter knowledge or image-specific software engineering. The dataset from this project was used subsequently to support the Radiological Society of North America (RSNA) 2017 Pediatric Bone Age Challenge, in which more than 230 teams competed.

\section{Satija U, Ramkumar B, Manikandan MS Automated ECG noise detection and classification system for unsupervised healthcare monitoring \\ IEEE J Biomed Health Inform 2018;22(3):722-32}

Electrocardiogram (ECG) signals are often corrupted by noise and artifacts, which can make it almost impossible to analyze the morphology and beat-to-beat interval of the signals. However, most existing ECG analysis systems are designed to handle relatively noise-free ECG signals. The authors proposed a novel framework to automatically detect, localize, and classify single and combined ECG noise. Evaluation on five benchmark ECG databases showed an average sensitivity of $99.12 \%$ and specificity of $98.56 \%$ to detect the presence of noise. The authors' approach achieved better noise detection and classification than the current state-of-the-art methods, and also accurately localized short bursts of noise that were present in ECG signals. 